\title{
On the Mathematical Models for Cancer Growth
}

\author{
Sorush Niknamian \\ $\mathrm{PhD}$ in Oncology, Cell and Molecular Biology, Department of Military Medicine, Liberty \\ University, United States of America \\ E-mail: sorush.niknamian@mycampus.apus.edu
}

\begin{abstract}
Cancer disease is the second cause of death in the United States and world-wide. Most Researchers estimate that 595,690 of American people will die from cancer at the end of the year 2017. That means 1,600 deaths/day approximately. Cancer in modern societies is commonly treated with the combination of organ surgery, chemotherapy and radiotherapy. Many kinds of diet strategies have been experimented. However, none of them have been particularly effective. Interestingly, there is some applied research suggesting that a very lowcarb ketogenic diet may help. According to Otto Warburg hypothesis, the cause of cancer is the change in the metabolism of mitochondrion in human cells. Low oxygen in tissues in combination with high blood glucose will change the cell respiration from aerobic to anaerobic which leads to fermentation type of respiration. In this research we have collected some useful mathematical models and describing the best model for cancer growth.
\end{abstract}

Keywords: Cancer; cancer growth; Mathematical Models; Warburg Hypothesis

\section{Materials and Methods}

\section{Exponential Growth Model}

One model that has been used to describe tumor growth is the exponential growth model [1].

$n(t)=N_{0} \exp (\lambda t)$

\section{Equation 1:}

The exponential does not describe the growth rate in vivo which slows are the tumor size increases.

\section{Gompertz Model}

Another model use to describe tumor dynamics is a Gompertz curve or Gompertz function. It is a type of mathematical model for a time series, where growth is slowest at the end of a time period $^{1}$.

$n(t)=N_{0} \exp \left[\ln \left(\frac{N_{\infty}}{N_{0}}[1-\exp (-b t)]\right)\right]$ 


\section{Equation 2:}

where $\mathrm{N} \infty$ is the plateau cell number which is reached at large values of $\mathrm{r}$ and the parameter $\mathrm{b}$ is related to the initial tumor growth rate.

Even with Gompertzian growth, a single set of growth parameters is insufficient to model the clinical data. Tumor cells almost certainly have different growth characteristics in different patients, and individual micrometastases within a single patient may also have different growth parameters.

\section{Model based on metabolic considerations}

To properly understand or derive a mathematical model for cancer growth, we must first understand the process of the ontogenetic development of an organism. This process is fueled by metabolism and follows a certain pattern which occurs primarily through cell division. In a recent paper, a mathematical model based on energy conservation was derived to model such growth and showed that regardless of the different masses and development times, all taxons share a common growth pattern [2]. It may be possible that cancer growth may be modeled in very much the same way. As the total energy that goes into an organism's development either goes into the maintenance of existing tissue or the creation of new tissue, we can express this as:

$B=\sum_{c}\left[N_{c} B_{c}+E_{c} \frac{d N_{c}}{d t}\right]$

\section{Equation 3:}

where, B is the energy that an organism uses while at rest. The variables, Bc and Nc are the metabolic rate for an individual cell and the number of cells in a particular organism respectively; the $\mathrm{NcBc}$ term represents the energy to maintain existing tissue. Ecis the energy needed to create new tissue from an individual cell.

We assume that variables $\mathrm{Ec}, \mathrm{Bc}$ and me all remain constant during an organism's growth and is pertinent to a particular type of organism. Thus the total mass of an organism, $\mathrm{m}$, can be determined from the mass of an individual cell and the number of cells, $\mathrm{m}=\mathrm{mcNc}$. By differentiating and substituting this into eq (3), we get

$\frac{d m}{d t}=B\left(\frac{m_{c}}{E_{c}}\right)-m\left(\frac{B_{c}}{E_{c}}\right)$

Equation 4

Given that $B=B_{0} m^{\frac{3}{4}}$

where B0 depends on a particular taxon, 
$\frac{d m}{d t}=B_{0} \frac{m_{c}}{E_{c}} m^{\frac{3}{4}}-\frac{B_{c}}{E_{c}} m$

Equation 5

As the B0, mc and Ec terms are constant, we can express the above equation succinctly as

$\frac{d m}{d t}=a m^{\frac{3}{4}}-b m$

Equation 6

where $\mathrm{a} \equiv \mathrm{B} 0 \mathrm{mc} / \mathrm{Ec}$ and $\mathrm{b} \equiv \mathrm{Bc} / \mathrm{Ec}$.

The 3/4 exponent is roughly the same for all organisms, whether they be mammals, birds, fish or plants. Thus the exponent describes the overall allometry of B from birth to maturity. As there is a tendency for natural selection to optimize energy transport, this has lead to the evolution of a fractal-like distribution network. This exponent is related to the scaling in the total number, $\mathrm{Nt}$ of capillaries ${ }^{3,4}$. As we already know, the total number of cells is related to the organism's mass. This exponent has the profound implication in that it sets limits on the growth of an organism. Thus the point in which an organism stops growing, i.e. $\mathrm{dm} / \mathrm{dt}=0$, we see that

$M=\left(\frac{a}{b}\right)^{4}=\left(\frac{B_{0} m_{c}}{B_{c}}\right)^{4}$

\section{Equation 7:}

where $\mathrm{M}$ is the symptotic maximum body size. Thus the variation on $\mathrm{M}$ among different species within a taxon is determined entirely by the cellular metabolic rate, $\mathrm{Bc}$, which scales to M-1/4. As cancer growth follows the same principles, blood and nutrients enter into and feed a tumor, we expect the same scaling principles to apply and thus by using this Universal Law for ontogenetic growth we hope to derive a similar universal law for cancer growth. As B0, mc and Ec are approximately constant, $a$ is independent of $\mathrm{M}$ and $\mathrm{b}=\mathrm{a} / \mathrm{M} 1 / 4$. We can thus rewrite eq (7) as

$$
\frac{d m}{d t}=a m^{\frac{3}{4}}\left[1-\left(\frac{m}{M}\right)^{\frac{1}{4}}\right]
$$

Equation 8

Solving this differential equation gives

$$
\left(\frac{m}{M}\right)^{\frac{1}{4}}=1-\left[1-\left(\frac{m_{0}}{M}\right)^{\frac{1}{4}}\right] e^{\left(-a t / 4 M^{\frac{1}{4}}\right)}
$$

\section{Equation 9:}


where $\mathrm{m} 0$ is the mass of the organism at birth $(\mathrm{t}=0)$. As a and $\mathrm{b}$ can be determined from fundamental parameters of a cell, a universal equation has been derived. We can see from the figures below that all growth curves follow the same path. We can infer that if similar considerations are made for cancerous cells, a similar growth curve will be obtained.
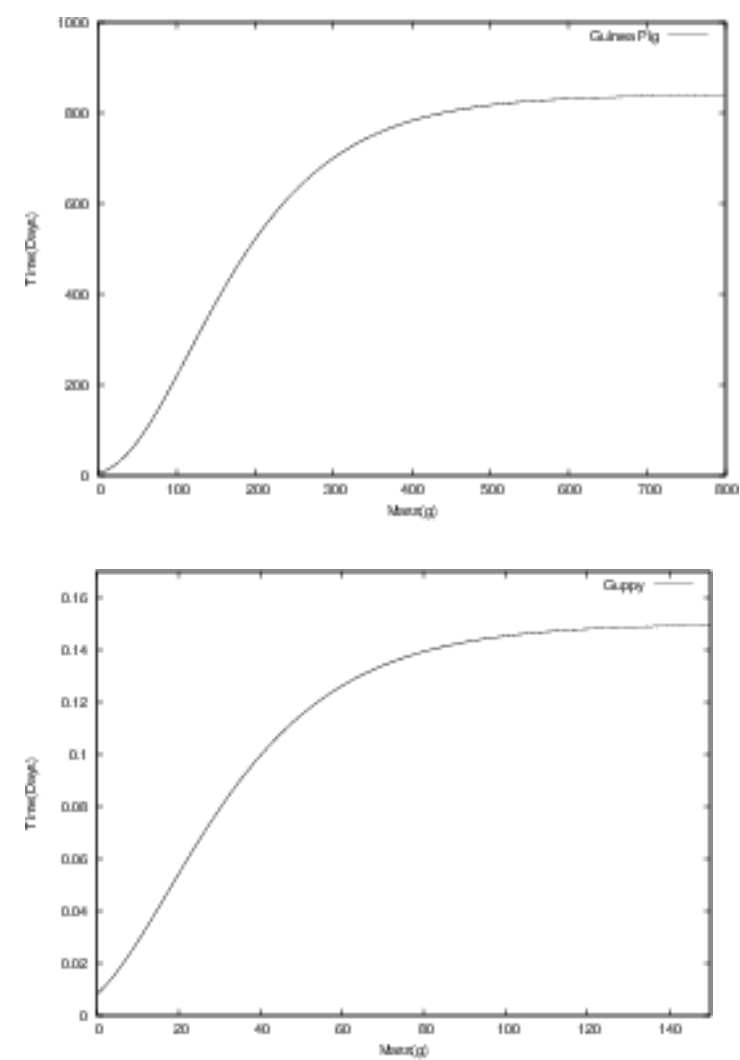

\section{Empirical Tests of Mathematical Models for Tumor Growth}

\section{Exponential Growth Law}

All tumors follow a standard growth pattern, growing fastest in the beginning and eventually reaching a maximum size. There are some weaknesses in the exponential growth model as it fails to model this behavior in vivo.

\section{Gompertz Law}

The Gompertz Model, while it does model the behavior as a tumor increases in size, it is not an empirical model. The model has too many variables to consider, such as types of cancers as well as environmental conditions. These may even vary considerably for patients with the same types of cancers [1].

\section{Universal Law}

The Universal Law model proposed by West, adequately describes the growth rates of organisms by taking energy considerations into account. This model was tested against empirical data ${ }^{5}$. As powerful as West's model for growth is, it only applies to organisms 
growing in unrestricted dietary conditions. Thus only fully replenished tumors will follow this universal growth curve. Differences in growth rates and saturation sizes of up to a factor of 500 were found in tumor spheroids cultured in media with different oxygen levels and glucose concentrations [6]. Thus deviations from West's Law depend on particular environmental conditions.

As a tumor reaches a certain size or volume, metastatic dissemination will occur [7]. As the rate of cell growth is dependent on its development phase, this may serve as a possible explanation why recurrent cancers grow at much slower rates than the primary tumors ${ }^{2}$. In this case, the residual clonogenic cells of the primary tumor generate cells from an older development phase and thus multiply at a slower rate.In general, the West model for ontogenetic growth of living organisms applies to the case of solid malignant tumors. The results fit a variety of in vitro and in vivo data [5].

\section{Growth Rates for Common Cancers}

Human tumor growth rates are approximately exponential when in vivo which means that the rate of growth depends on developmental stage ${ }^{8}$. Tumor growth follows an exponential growth, as seen in the exponential, Gompertz and universal law models which means rate changes over time. To best describe the growth rate of a tumor, it is best to describe the growth rate in terms of doubling time, or the time it takes for the population of cells to reach twice its sizeAs there is a lack of clinical data at non-symptomatic stages, it can be assumed that two to three decades can elapse between the first carcinogenic stimulus and the emergence of the neoplasm [12]. Typical doubling times for a tumor is expected to be between 60 days for very aggressive tumors and 100 days for non-aggressive tumors. [9-11]

\section{Discussions and Results}

While there isn't one specific model that can adequately describe any one tumor, each model does highlight certain aspects of tumor kinetics. The Gompertz model, for example, highlights that a tumors rate of growth is greatest at the beginning stages; the point when there are no means to detect them clinically. The model also predicts that as the tumor grows, its growth rate slows down. Despite predicting these behaviors there is no real basis for explaining these attributes as this model is an empirical law. The West law on the other hand is a fundamental law as it is based on basic physical laws; the conservation of energy. From this law we can intuit and explain certain observed behaviors, for one, that there would be a maximum size that a tumor will attain. This maximum size is due the 'fractal' pattern of the veins that feed and nourish the tumor; as the veins bifurcates there comes a point that the vein is too small to adequately supply nutrients to the cancerous cells. As the West Model also says that the growth rate changes with developmental stage, it explains why the growth rates of tumors that have metastasized don't grow as rapidly as the tumorous cells from which they originated.

\section{Conclusions}

The development of tumor models is important as they offer a way to better understand the growth kinetics of malignant tumors which may lead to the development of successful treatment strategies. [13-15] Based on all the models discussed above, Model based on 
metabolic considerations may be the most useful models for showing cancer growth and the proof of cancer as a metabolic disease. Although main mathematical models have been discussed, there is a need for further investigation into future research to reach the most universal model to describe all cancer growth as a one unique mathematical model.

\section{References}

1 Yorke, E. D., Fuks, Z., Norton, L., Whitmore, W. \& Ling, C. C. Modeling the Development of Metastases from Primary and Locally Recurrent Tumors: Comparison with a Clinical Data Base for Prostatic Cancer. Cancer Research 53, 2987-2993 (1993).

2 West, G. B., Brown, J. H. \& Enquist, B. J. A general model for ontogenetic growth. Nature 413 ,

628-631, doi:http://www.nature.com/nature/journal/v413/n6856/suppinfo/413628a0_S1.html (2001).

3 West, G. B., Woodruff, W. H. \& Brown, J. H. Allometric scaling of metabolic rate from molecules and mitochondria to cells and mammals. Proc. Natl. Acad. Sci. U. S. A. 99, 24732478, doi:10.1073/pnas.012579799 (2002).

4 Delsanto, P. P. et al. Growth model for multicellular tumor spheroids. Appl. Phys. Lett. 85, 4225-4227, doi:10.1063/1.1812842 (2004).

5 Guiot, C., Degiorgis, P. G., Delsanto, P. P., Gabriele, P. \& Deisboeck, T. S. Does tumor growth follow a 'universal law'? (2003).

6 Freyer, J. P. \& Sutherland, R. M. Regulation of Growth Saturation and Development of Necrosis in EMT6/Ro Multicellular Spheroids by the Glucose and Oxygen Supply. Cancer Research 46, 3504-3512 (1986).

7 Romsdahl, M. D., Chu, E. W., Hume, R. \& Smith, R. R. The time of metastasis and release of circulating tumor cells as determined in an experimental system. Cancer 14, 883-888, doi:10.1002/1097-0142(199007/08)14:4<883::aid-cncr2820140426>3.0.co;2-8 (1961).

8 Mackillop, W. J. The growth kinetics of human tumours. Clinical Physics and Physiological Measurement 11, 121 (1990).

9 Spratt, J. S., Jr. \& Spratt, T. L. RATES OF GROWTH OF PULMONARY METASTASES AND HOST SURVIVAL. Annals of surgery 159, 161-171 (1964).

10 Steele, J. D. \& Buell, P. Asymptomatic solitary pulmonary nodules. Host survival, tumor size, and growth rate. The Journal of thoracic and cardiovascular surgery 65, 140-151 (1973).

11 Collins, V. P., Loeffler, R. K. \& Tivey, H. Observations on growth rates of human tumors. The American journal of roentgenology, radium therapy, and nuclear medicine 76, 988-1000 (1956).

12 Tubiana, M. The growth and progression of human tumors: Implications for management strategy. Radiother. Oncol. 6, 167-184, doi:Doi: 10.1016/s0167-8140(86)80151-7 (1986). 
13 Chis, O. \& Opris, D. Mathematical analysis of stochastic models for tumor-immune systems. (2009).

14 Chignola, R. et al. Forecasting the growth of multicell tumour spheroids: implications for the dynamic growth of solid tumours. Cell Proliferation 33, 219-229, doi:10.1046/j.13652184.2000.00174.x (2000).

15 Norton, L. A Gompertzian Model of Human Breast Cancer Growth. Cancer Research 48, 7067-7071 (1988). 